\title{
TRAVELLING COMBUSTION WAVES IN A POROUS MEDIUM. PART I-EXISTENCE*
}

\author{
J. NORBURY $\dagger$ AND A. M. STUART $\ddagger$
}

\begin{abstract}
A one-space-dimensional, time-dependent model for travelling combustion waves in a porous medium is analysed. The key variables are the temperature of the solid medium and its density and the temperature of the gaseous phase and its density. The key parameters $\mu, \lambda$ and $a$ are related (respectively) to the driving gas velocity, the specific heat of the combustible solid and the ratio of consumption of oxygen to that of solid. The regions of existence of the different types of combustion waves are found in $(\mu, \lambda)$ parameter space, with $a=0$. The types of combustion wave are classified by the switch mechanism that turns off the combustion, which occurs over a finite, but unknown, interval. Because the model is linear outside the combustion zone, the eigenvalue problem governing the existence of travelling waves may be reformulated as a two-point free boundary problem on a finite domain. Existence and nonexistence theorems are established for this unusual bifurcation problem.
\end{abstract}

Key words. combustion, travelling waves, existence

AMS(MOS) subject classifications. 34B15, 35B32, 80A30

1. Introduction. In this paper we analyse a one-dimensional, time-dependent model for porous medium combustion. The equations form a simplified version of the model proposed by Lawson and Norbury in [3] and the details of the simplifications may be found in [5]. We pose the equations on an infinite domain and seek travelling wave solutions to the problem. The equations are linear outside a finite region of space in which combustion occurs and so we reduce the eigenvalue problem governing the existence of travelling waves to a two-point free boundary problem. By applying local bifurcation theory we prove the existence of a branch of nontrivial solutions to this free boundary problem. We examine the structure of the solutions on this branch (shown by Fig. 1 in $\S 6$ ) by a combination of numerical and series expansion techniques. In particular we demonstrate the importance of the parameters representing the inlet gas velocity and the specific heat of the combustible solid in determining the existence and form of the travelling wave solutions.

A wide variety of combustion phenomena have been modelled by idealising a propagating combustion zone as a travelling wave solution of an appropriate set of model equations. In particular, Matkowsky and Sivashinsky [4] model solid fuel combustion in such a fashion. The situation which we analyse is somewhat different from this because, while we also consider the combustion of a solid, the medium is assumed to be porous. This affects the modelling fundamentally: first, we must include a description of the conservation of gas mass and energy, and second, the nature of the reaction rate differs substantially from that in solid fuel combustion because the reaction rate is limited by the ability of the gaseous reactants to diffuse between the gas mainstream and the reaction sites.

The derivation of the model which we analyse here is described in [5]. The four dependent variables are $\sigma, u, w$ and $g$ which represent, respectively, the heat capacity of the solid, the solid temperature, the gas temperature and a quantity proportional

\footnotetext{
* Received by the editors August 11, 1986; accepted for publication (in revised form) April 23, 1987.

$\uparrow$ Mathematical Institute, 24-28 St. Giles, Oxford OX1 3LB, England.

\$Oxford University Computing Laboratory, Numerical Analysis Group, 8-11 Keble Road, Oxford OX1 3QD, England. The work of this author was supported by the Science and Engineering Research Council of the United Kingdom.
} 
to the product of the oxygen concentration and the gas temperature. The heat capacity is a linear function of the concentration of combustible solid, and thus the equation for $\sigma$ governs the concentration of combustible solid. The parameter $\mu$ is proportional to the inlet gas velocity, while the parameter $\lambda$ is linearly related to the specific heat of the combustible solid. The parameter $a$ is proportional to the ratio of the rate of consumption of oxygen to that of solid.

The equations are

$$
\begin{aligned}
& \frac{\partial \sigma}{\partial t}=-\lambda r \\
& \sigma \frac{\partial u}{\partial t}=\frac{\partial^{2} u}{\partial z^{2}}+w-u+r \\
& \mu \frac{\partial w}{\partial z}=u-w \\
& \frac{\partial g}{\partial z}=-\frac{a}{\mu} r .
\end{aligned}
$$

Here the reaction rate $r$ is given by

$$
r=\mu^{1 / 2} H(g) H(\sigma-\tau) H\left(u-u_{c}\right) f(w) g,
$$

where $H(u)$ is defined to be zero when $u \leqq 0$ and unity when $u>0$. The function $f(w)$ is usually defined to be proportional to $w^{2}$; however, much of the analysis applies to a general positive function $f$. We now make the following definition. For both the partial differential equation system (1.1)-(1.8) and the ordinary differential equation system (2.1)-(2.6) we define the burning zone to be the interval in $z$ space in which the reaction rate $r$ satisfies $r>0 . \tau \in[0,1)$.

The boundary conditions for (1.1)-(1.5) are taken to be

$$
u( \pm \infty, t)=u_{a}, \quad w(-\infty, t)=u_{a} \quad \text { and } \quad g(-\infty, t)=g_{a} .
$$

The ambient temperature $u_{a}$ is typically much smaller than $u_{c}$, the critical switching temperature.

Throughout this paper we will take $a \equiv 0$ and redefine $f(w)$ to absorb the factor $g_{a}$. In so doing we are describing combustion processes in which either the rate of oxygen depletion is slow or in which the initial concentration of oxygen is high. This will not affect much of the analysis, although for $\mu \ll 1$ it will clearly be unrealistic, by virtue of (1.4). We discuss this matter further in $\S 6$.

The model defined by (1.2)-(1.6) does not include the effect of radiation in the solid energy equation (1.2). However, the qualitative nature of the results in this paper are not affected if we include this effect. In fact, the results of $\S \S 2-4$ are completely unchanged. The details of the algebra involved in the power series expansions in $\S 5$ will, however, be modified, as will the quantitative nature of the numerical results in $\S 6$.

In $\S 2$ we derive the eigenvalue problem governing the existence of travelling wave solutions to the partial differential equations (1.1)-(1.8). In $\S 3$ we show how this problem may be converted to a two-point free boundary problem. In $\S 4$ we prove two theorems on the existence of solutions to the free boundary problem. In $\S 5$ we employ perturbation series expansions to determine the form of the solution in various parameter limits. In $\S 6$ we describe some numerical results which extend our understanding of the solution behaviour away from regions in which the expansions are valid. 
Because of the form of the reaction term (1.5) there is no bifurcation of travelling combustion waves from the trivial state of no burning. However, we demonstrate a technique for obtaining a local analytical description of the global solution branch representing combustion. We do this by stretching an artificial trivial solution with a burning zone of zero length onto a solution with a burning zone of finite length. This rescaling, which relies on the nature of the step function in the reaction rate (2.5), enables the techniques of local bifurcation theory to be applied. The resultant approximations in the neighbourhood of the bifurcation point then provide starting points for a global analysis of the solution branch by use of numerical techniques.

In summary, the analytical, numerical and series expansion results in this paper support the conjecture that Figs. 1 and 3 (see $\S 6$ ) define the generic behaviour of solution branches representing travelling wave solutions of (1.1)-(1.6). In particular we find that increasing the inlet gas velocity or the solid specific heat leads to regimes in which steady combustion cannot occur. Furthermore, we find that for solutions to exist, we require $\lambda>0$; thus it is necessary to allow for changes in the overall solid heat capacity of the medium in order that combustion may occur. This is in contrast with the theory of homogeneous combustion, where it is frequently assumed that the concentration of reactant is so small, relative to the concentration of inerts, that the overall physical properties of the medium do not change as the combustion reaction takes place. The stability of the steady travelling wave solutions will be discussed in Part II of this paper [7].

2. The governing eigenvalue problem. We make the transformation of independent variable $x=z-c t$ in (1.1)-(1.3) and write ' $\equiv d / d x$. We also define $Q(x)=c \sigma(z, t)$, $W(x)=w(z, t)$ and $U(x)=u(z, t)$. The equations become

$$
\begin{aligned}
& Q^{\prime}=\lambda r, \\
& \mu W^{\prime}=U-W, \\
& U^{\prime \prime}+Q U^{\prime}+W-U+r=0 .
\end{aligned}
$$

The reaction rate $(1.5)$ is now

$$
r=H(Q-\tau c) H\left(U-u_{c}\right) \mu^{1 / 2} f(W) .
$$

The boundary conditions (1.6) and (1.7) give us

$$
U( \pm \infty)=u_{a} \text { and } W(-\infty)=u_{a} .
$$

By assuming $U \rightarrow u_{a}\left(<u_{c}\right)$ as $x \rightarrow \infty$ so that no reaction occurs at $x=+\infty$, we deduce that the solid concentration there is given by its initial value, so that

$$
Q(\infty)=c
$$

The set of equations (2.1)-(2.6) forms a nonlinear eigenvalue problem for the wave velocity $c$ and the solution is a heteroclinic orbit in the four-dimensional $\left(Q, W, U, U^{\prime}\right)$ phase-space of the problem. We now examine the structure of the burning zone for the case of travelling waves. We claim that there are two distinct classes of travelling wave dependent upon the nature of the switch from $r=0$ to $r \neq 0$. We describe these.

(i) $A(U, U)$ wave. This is the case in which both ends of the burning zone are determined by points at which $U=u_{c}$. In other words the reaction switches off because the solid temperature becomes too low.

(ii) $A(Q, U)$ wave. In this case the left-hand end of the burning zone occurs because of solid exhaustion $(Q=\tau c)$, whereas the right-hand end is marked by the point at which the solid temperature falls below $u_{c}$. 
Because of the boundary condition (2.6) on $Q(\infty)$ no other switching combinations are possible. If $a>0$ then another switching mechanism becomes possible since oxygen exhaustion becomes possible at the right-hand end of the burning zone. This case is discussed further in $\S 6$.

Examination of the ordinary differential equation system (2.1)-(2.7) shows that for $r \equiv 0$ the only solution is the trivial one

$$
U=W=u_{a} \text { and } Q=c .
$$

This solution exists for all values of the wave velocity $c$ and represents the ambient state of no burning.

Nontrivial solution branches are necessarily disjoint from the trivial branch of solutions since any nontrivial solution satisfies $\|U\|_{\infty} \geqq u_{c}>u_{a}$. Thus it is not immediately clear that we may obtain any local analytical description of nontrivial solution branches. Our approach is to reduce the problem of finding travelling waves to a shooting problem on a finite domain and then to seek solutions characterised by $\|U\|_{\infty} \sim u_{c}$. In so doing we are motivated by the study of nonlinear parabolic equations with discontinuous forcing terms [6].

3. Reduction to a shooting problem. First we derive the shooting problem appropriate to case (i), the $(U, U)$ switch. We define the burning zone to be $0<x<L$. Thus for $x \notin(0, L), r=0$ and the governing equations are linear and integrable.

$-\infty<x<0$.

The solution of $(2.1)-(2.7)$ for $-\infty<x<0$ is

$$
\begin{aligned}
& U(x)=u_{a}+\left(u_{c}-u_{a}\right) e^{\alpha x}, \\
& W(x)=u_{a}+\left(u_{c}+u_{a}\right) e^{\alpha x} /(1+\mu \alpha), \\
& Q(x)=q_{L} .
\end{aligned}
$$

Here $q_{L}$ is an unknown, to be determined as part of the solution, and $\alpha$ is the positive root of a quadratic and given by

$$
2 \alpha=-\left(q_{L}+\mu^{-1}\right)+\left[\left(q_{L}+\mu^{-1}\right)^{2}-4\left(q_{L} \mu^{-1}-1\right)\right]^{1 / 2} .
$$

To ensure that a positive root $\alpha$ exists, so that $U$ and $W$ are bounded at $x=-\infty$, we require $q_{L}<\mu$.

$L<x<\infty$.

In $L<x<\infty$, (2.1)-(2.6) integrate to give

$$
\begin{aligned}
& U(x)=u_{a}+B\left(u_{c}-u_{a}\right) e^{\beta_{+}(x-L)}+(1-B)\left(u_{c}-u_{a}\right) e^{\beta_{-}(x-L)}, \\
& W(x)=u_{a}+\frac{B\left(u_{c}-u_{a}\right)}{\left(\mu \beta_{+}+1\right)} e^{\beta_{+}(x-L)}+\frac{(1-B)\left(u_{c}-u_{a}\right)}{\left(\mu \beta_{-}+1\right)} e^{\beta_{-}(x-L)}, \\
& Q(x)=c .
\end{aligned}
$$

Here $B$ is an unknown, to be determined, and $\beta_{+}$and $\beta_{-}$are the roots of

$$
\beta^{2}+\left(c+\mu^{-1}\right) \beta+\left(c \mu^{-1}-1\right)=0 \text {. }
$$

Hence

$$
2 \beta_{ \pm}=-\left(c+\mu^{-1}\right) \pm\left[\left(c+\mu^{-1}\right)^{2}-4\left(c \mu^{-1}-1\right)\right]^{1 / 2} .
$$

To ensure that we have two negative roots (which we need to determine $c$ ) we require $\mu<c$. 
We may now write down the governing equations on an unknown, but finite, domain $(0, L)$ with boundary conditions given by the solutions found for $x \notin(0, L)$.

$$
0<x<L
$$

$$
\begin{aligned}
& Q^{\prime}=\lambda \mu^{1 / 2} f(W), \\
& U^{\prime \prime}+Q U^{\prime}+W-U+\mu^{1 / 2} f(W)=0, \\
& W^{\prime}=(U-W) / \mu .
\end{aligned}
$$

The ordinary differential equations (2.1)-(2.6) imply continuity of $Q, U, U^{\prime}$ and $W$ at $x=0$ and $x=L$. Thus, at $x=0$, we obtain

$$
\begin{aligned}
& Q(0)=q_{L}(\alpha), \\
& U(0)=u_{c}, \\
& U^{\prime}(0)=\left(u_{c}-u_{a}\right) \alpha, \\
& W(0)=u_{a}+\frac{\left(u_{c}-u_{a}\right)}{1+\mu \alpha} .
\end{aligned}
$$

Here $q_{L}(\alpha)$ is determined implicitly by (3.4). At $x=L$, we obtain

$$
\begin{aligned}
& Q(L)=c \\
& U(L)=u_{c}, \\
& U^{\prime}(L)=B\left(u_{c}-u_{a}\right) \beta_{+}+(1-B)\left(u_{c}-u_{a}\right) \beta_{-}, \\
& W(L)=u_{a}+\frac{B\left(u_{c}-u_{a}\right)}{\left(\mu \beta_{+}+1\right)}+\frac{(1-B)\left(u_{c}-u_{a}\right)}{\left(\mu \beta_{-}+1\right)} .
\end{aligned}
$$

Equations (3.9)-(3.19) form a two-point boundary value problem involving a fourth order system of ordinary differential equations (for $Q, W, U$ and $U^{\prime}$ ) and four unknown constants; these constants are $\alpha, L, c$ and $B$. Notice that the system (3.9)-(3.15) is properly posed as an initial value problem, so that the extra boundary conditions at $x=L$ determine the four constants. Since one of the unknowns is $L$, the length of the domain, the problem is of free-boundary type. It is also nonlinear via the coupling of the differential equations and through the nonlinear dependence of the $\beta$ 's on $c$. In order that a solution of this boundary value problem is a true bounded travelling wave solution we must also impose the constraints that any solution found must satisfy

$$
q_{L}=Q(0)<\mu<Q(L)=c .
$$

Also, since we are considering a $(U, U)$ switch, we require

$$
\tau c<Q(0) \text {. }
$$

Note that $0 \leqq \tau<1$.

We can eliminate $B$ and $c$ from the boundary value problem in the following way. Using (3.9), (3.16), (3.18) and (3.19) we may show that

$$
U^{\prime}(L)+\left(u_{c}-u_{a}\right) Q(L)-\mu\left[W(L)-u_{a}\right]=0 .
$$

Thus we now define the following one-parameter shooting problem.

Definition of shooting problem 1 for the $(U, U)$ switch. Integrate (3.9)-(3.11), subject to initial conditions (3.12)-(3.15) until $x=L: U(L)=u_{c}$. The correct value of $\alpha$ is found when (3.22) is satisfied. 
Remarks on shooting problem 1. (i) Notice that when the shooting problem has been solved the correct values of $c$ and $B$ may be determined directly from the final values of $Q$ and $U^{\prime}$ by using (3.16) and (3.18).

(ii) The solution of the shooting problem only represents a true travelling wave solution if constraints (3.20) and (3.21) are satisfied.

When $\tau=0$ (which corresponds to a combustion process in which the ash has zero heat capacity) a similar reduction to a one-parameter shooting problem is possible for case (ii), the $(Q, U)$ switch. The only difference from the shooting problem 1 is that the initial conditions become

$$
\begin{aligned}
& Q(0)=0, \\
& U(0)=u_{b}, \\
& U^{\prime}(0)=\left(u_{b}-u_{a}\right) \alpha, \\
& W(0)=u_{a}+\left(u_{b}-u_{a}\right) /(\mu \alpha+1) .
\end{aligned}
$$

Now $\alpha$ is known and given, from (3.4) with $q_{L}=0$. The shooting parameter is $u_{b}$.

Definition of shooting problem 2 for the $(Q, U)$ switch. Integrate (3.9)-(3.11), subject to initial conditions (3.23)-(3.26) until $x=L: U(L)=u_{c}$. The correct value of $u_{b}$ is found when (3.22) is satisfied.

Remarks on shooting problem 2. (i) The solution only represents a true travelling wave solution if certain constraints, namely (3.20) and $u_{b} \geqq u_{c}$, are satisfied to ensure that we are in the regime of a $(Q, U)$ switch.

(ii) We can define a two-parameter shooting problem in the (more realistic) case $\tau \neq 0$. The two-parameters are then $\alpha$ and $u_{b}$. We return to this more general shooting problem in $\S 5$.

4. Existence theorems. We now prove two theorems about the existence of nontrivial solutions to the travelling wave problem. The following theorem reflects an energy balance, relating the amount of solid consumed to the amount of heat produced.

THEOREM 4.1. Nontrivial solutions to the nonlinear eigenvalue problem defined by (2.1)-(2.6) can only exist for $0<\lambda<\lambda_{c}$, where $\lambda_{c}=\left(u_{c}-u_{a}\right)^{-1}$.

Proof. Integrate (2.3), using (2.2), from $-\infty<x<\infty$. This gives

$$
U^{\prime}(\infty)-U^{\prime}(-\infty)+\int_{-\infty}^{+\infty} Q U^{\prime} d x+\mu[W(\infty)-W(-\infty)]+\int_{-\infty}^{+\infty} r d x=0,
$$

where $r$ is defined by (2.4). Since we know that $U^{\prime}( \pm \infty)=0$ and $W( \pm \infty)=u_{a}$ for travelling wave solutions, we have

$$
\int_{-\infty}^{+\infty} Q U^{\prime} d x+\int_{-\infty}^{+\infty} r d x=0
$$

Integrating by parts, using (2.1), gives us

$$
u_{a}[Q(\infty)-Q(-\infty)]+\int_{-\infty}^{+\infty}(1-\lambda U) r d x=0
$$

But, we may now eliminate $Q(\infty)-Q(-\infty)$, again using equation (2.1), to obtain

$$
\int_{-\infty}^{+\infty}\left[1+\lambda\left(u_{a}-U\right)\right] r d x=0
$$

Since $r=0$ for $x \notin(0, L)$ we finally have

$$
\int_{0}^{L}\left[1+\lambda\left(u_{a}-U\right)\right] \mu^{1 / 2} f(W) d x=0
$$


Thus any travelling wave solution must satisfy (4.3). Since $f(W)>0, U(x)>u_{c}>u_{a}$ for $x \in(0, L)$ and $L>0$ for nontrivial solutions, the result follows.

We now examine shooting problem 1 in greater detail. In what follows it is advantageous to pose the shooting problem on a fixed domain. Thus we rescale the independent variable $x$ by defining $\bar{y}=x / L$. Applying this transformation to (3.9)(3.11) we obtain

$$
\begin{aligned}
& Q_{\bar{y}}=\lambda \mu^{1 / 2} L f(W), \\
& U_{\bar{y} \bar{y}}+L Q U_{\bar{y}}+L^{2}(W-U)+\mu^{1 / 2} L^{2} f(W)=0, \\
& W_{\bar{y}}=L(U-W) / \mu .
\end{aligned}
$$

Boundary conditions (3.12), (3.13) and (3.15) at $\bar{y}=0$ are unchanged. Boundary condition (3.14) becomes

$$
U_{\bar{y}}(0)=\left(u_{c}-u_{a}\right) L \alpha .
$$

The matching conditions which implicitly determine $L$ and $\alpha$ are (3.17) and (3.22), which become

$$
U(1)=u_{c} \quad \text { and } \quad L \int_{0}^{1}\left[1+\lambda\left(u_{a}-U\right)\right] \mu^{1 / 2} f(W) d x=0 .
$$

We have used (4.1) rather than (3.22), since they may be shown to be equivalent, by applying the method of Theorem 4.1 on the finite length burning zone.

Examination of the boundary value problem defined by (4.2)-(4.6) and (3.12), (3.13) and (3.15) shows that it possesses the family of trivial solutions

$$
L=0, \quad \alpha=\bar{\alpha}, \quad U \equiv u_{c}, \quad Q \equiv q_{L}(\bar{\alpha}) \quad \text { and } \quad W \equiv u_{a}+\left(u_{c}-u_{a}\right) /(1+\mu \alpha)
$$

parameterised by $\bar{\alpha} \in \mathbb{R}$. Since the transformation of variable $\bar{y}=x / L$ is only valid for nonzero $L$, these solutions do not correspond to true travelling wave solutions. However, we may now apply the techniques of local bifurcation theory [1] to determine the possible location of bifurcation points on the trivial branch(es) of solutions into nontrivial solutions. Because of constraints (3.20) and (3.21), the only trivial solution of any interest to us is the one with $\bar{\alpha}=0$, so that $Q \equiv \mu$. This is because it is the only trivial solution which, when perturbed by an arbitrarily small amount, can satisfy both (3.20) and (3.21). The following theorem examines the possibility of bifurcation from this particular trivial solution.

THEOREM 4.2. Consider the trivial solution (4.7) of shooting problem 1 given by $\bar{\alpha}=0$, namely

$$
Q \equiv \mu, \quad U \equiv u_{c}, \quad W \equiv u_{c}, \quad L=0 \quad \text { and } \quad \alpha=0 .
$$

Then a necessary condition for bifurcation into nontrivial solutions of shooting problem 1 is $\lambda=\lambda_{c}$.

Proof. Let $\psi=(\psi, \theta, \phi, \xi, \eta)$ represent the linearisation of $(Q, U, W, L, \alpha)$ about the trivial solution (4.8). We consider the solution and its linearisation as elements of the Banach space $C^{1}(0,1) \times C^{2}(0,1) \times C^{1}(0,1) \times \mathbb{R} \times \mathbb{R}$. Then $\psi$ satisfies

$$
\psi_{\bar{y}}=\xi \lambda \mu^{1 / 2} f\left(u_{c}\right) \text { and } \theta_{\bar{y} \bar{y}}=\phi_{\bar{y}}=0 \text {, }
$$

where the initial conditions are

$$
\begin{aligned}
& \psi(0)=-\left(1+\mu^{2}\right) \eta, \\
& \theta(0)=\theta_{\bar{y}}(0)=0, \\
& \phi(0)=-\left(u_{c}-u_{a}\right) \mu \eta
\end{aligned}
$$


The matching conditions are

$$
\theta(1)=0, \quad \xi \int_{0}^{1}\left[1+\lambda\left(u_{a}-u_{c}\right)\right] \mu^{1 / 2} f\left(u_{c}\right) d y=0 .
$$

This linear problem for $\psi$ defines the Fréchet derivative of the shooting problem defined by (4.2)-(4.6) and (3.12), (3.13) and (3.15), with respect to the trivial solution (4.8). In the case $\lambda \neq \lambda_{c}\left(=\left(u_{c}-u_{a}\right)^{-1}\right)$ the null-space of the Fréchet derivative is one-dimensional and spanned by the eigenfunction

$$
(\psi, \theta, \phi, \xi, \eta)=\left(-\left(1+\mu^{2}\right), 0,-\left(u_{c}-u_{a}\right) \mu, 0,1\right) .
$$

This eigenfunction corresponds to bifurcation into the trivial branch of solutions (4.7) parameterized by $\bar{\alpha}$. However, in the case $\lambda=\lambda_{c}$, the null-space of the Fréchet derivative is two-dimensional and spanned by (4.10) and a second eigenfunction

$$
(\psi, \theta, \phi, \xi, \eta)=\left(\lambda_{c} \mu^{1 / 2} f\left(u_{c}\right) \bar{y}, 0,0,1,0\right) .
$$

This extra eigenfunction derives from the noninvertibility of expression (4.9) for $\xi$ at $\lambda=\lambda_{c}$. Consequently, $\lambda=\lambda_{c}$ is the only point at which bifurcation into nontrivial solutions can occur.

Remarks on Theorem 4.2. (i) Since the null-space of the Fréchet derivative is even-dimensional we have not determined a sufficient condition for bifurcation at $\lambda=\lambda_{c}$ [1]. However, in $\S 5$, we prove that there is a nontrivial branch of solutions of shooting problem 1 bifurcating from the trivial solution (4.8) at $\lambda=\lambda_{c}$, by constructing a power series expansion for the nontrivial solution.

(ii) The two-dimensional null-space of the Fréchet derivative at $\lambda=\lambda_{c}$ provides the necessary degrees of freedom for bifurcation into nontrivial travelling wave solutions, since it allows the possibility of solutions of shooting problem 1 that satisfy (3.20). We return to this point in Theorem 5.1.

5. Approximations by power series. In this section we examine the asymptotic form of the travelling wave solutions in the two separate limits $\lambda \rightarrow \lambda_{c}$ and $\mu \rightarrow 0$. We also examine the case $\lambda \rightarrow \lambda_{c}, \mu \rightarrow 0$ for a limiting value of $\left(\lambda-\lambda_{c}\right) / \mu$ which is of particular interest.

Power series solution for fixed $\mu>0$ and $\left(\lambda_{c}-\lambda\right) \rightarrow 0$. Here we construct a regular power series expansion of the solution of shooting problem 1 which bifurcates from the trivial solution at $\lambda=\lambda_{c}$. Since these solutions are necessarily of small sup-norm relative to the trivial solution (4.12) of shooting problem 1 , we deduce that they are characterised by $L \ll 1$. Thus we rescale (4.2)-(4.6) and (3.12), (3.13) and (3.15) by setting $L=\varepsilon$, where $\varepsilon \ll 1$.

The appropriate form of the power series expansion is then

$$
\begin{aligned}
& Q=\mu+\varepsilon Q_{1}+\varepsilon^{2} Q_{2}+\cdots, \\
& U=u_{c}+\varepsilon^{2} U_{2}+\varepsilon^{3} U_{3}+\cdots, \\
& W=u_{c}+\varepsilon W_{1}+\varepsilon^{2} W_{2}+\cdots, \\
& \alpha=\varepsilon \alpha_{1}+\varepsilon^{2} \alpha_{2}+\cdots, \\
& \lambda=\lambda_{c}-\varepsilon^{2} \lambda_{c 2}+\cdots
\end{aligned}
$$

To first order we obtain in terms of the original variable $x$, for $0<x<\varepsilon$, 


$$
\begin{aligned}
& Q(x)=\mu+\alpha_{1}\left[2 x-\left(1+\mu^{2}\right) \varepsilon\right]+O\left(\varepsilon^{2}\right), \\
& U(x)=u_{c}+\frac{\alpha_{1}}{\lambda_{c}}(\varepsilon-x) x+O\left(\varepsilon^{3}\right) \\
& W(x)=u_{c}-\frac{\mu \alpha_{1} \varepsilon}{\lambda_{c}}+O\left(\varepsilon^{2}\right)
\end{aligned}
$$

Here $\alpha_{1}$ is given by

$$
\alpha_{1}=\frac{1}{2} \mu^{1 / 2} f\left(u_{c}\right) \lambda_{c}
$$

the value of $\lambda_{c 2}$ is determined at higher order.

Since this regular convergent series expansion always satisfies the matching conditions (4.9) and (4.10), by suitable choice of $\alpha$ and $\left(\lambda-\lambda_{c}\right)$ expanded as series in $\varepsilon$, this demonstrates the existence of a branch of nontrivial solutions of shooting problem 1 bifurcating from $\lambda=\lambda_{c}$. Using the power series expansion of this bifurcating solution we may now prove the following result.

THEOREM 5.1. For $\mu<1$ there exists a nontrivial branch of travelling wave solutions of the problem defined by (2.1)-(2.6) bifurcating from $\lambda=\lambda_{c}$. For $\mu>1$ there does not.

Proof. We showed in $\S 3$ that there is a one-to-one correspondence between solutions to the travelling wave problem and shooting problems 1 and 2, provided that certain constraints on the solutions of the shooting problems are satisfied. We have demonstrated, by means of a power series expansion, the existence of a nontrivial branch of solutions to shooting problem 1 for all $\mu$. Thus we must determine when constraints (3.20) and (3.21) are satisfied (since these are the constraints that ensure that solutions of shooting problem 1 are also solutions of the travelling wave problem).

From (5.1) it is clear that (3.21) is satisfied for sufficiently small $\varepsilon$, since $0 \leqq \tau<1$ and $c=Q(L)=\mu+O(\varepsilon)$. Using the expression (5.4) for $\alpha_{1}$ we obtain

$$
Q\left(\varepsilon L_{1}\right)=\mu+\frac{1}{2} \lambda_{c} \mu^{1 / 2} f\left(u_{c}\right)\left(1-\mu^{2}\right) \varepsilon+O\left(\varepsilon^{2}\right)
$$

Hence, for sufficiently small $\varepsilon$, the constraint $\mu<Q(L)$ is satisfied if $\mu<1$, whereas for $\mu>1$ it is not.

Form of solutions for $0<\lambda<\lambda_{c}$ and $\mu \rightarrow 0$. Examination of the approximate form of solutions of shooting problem 1 in this regime leads to the conclusion that $Q(0) \sim$ $-\mu^{1 / 4} \bar{\alpha}$, where $\bar{\alpha}$ is a positive number. Hence these solutions cannot satisfy constraint (3.21). Thus we expect that, for fixed $0<\lambda \leqq \lambda_{c}$ and $\mu \rightarrow 0$, we are in a parameter regime where a $(Q, U)$ switch operates and so we consider shooting problem 2 . Furthermore it can be shown that in this parameter regime solutions do not exist unless $\tau=0$, so we consider this case.

Analysis shows that consistent scalings of the variables in the case $\mu \ll 1$ are

$$
Q \sim \mu^{1 / 4} Q_{0}, \quad L \sim \mu^{-1 / 4} L_{0}, \quad U \sim U_{0}, \quad W \sim W_{0} \quad \text { and } \quad u_{b} \sim U_{b 0},
$$

where a subscript zero indicates an order one quantity. If we now define $y=\mu^{1 / 4} x$ then with the scalings described above shooting problem 2 gives, to leading order,

$$
\begin{aligned}
& Q_{0 y}=\lambda f\left(U_{0}\right), \\
& U_{0 y y}+Q_{0} U_{0 y}+f\left(U_{0}\right)=0, \\
& W_{0}=U_{0},
\end{aligned}
$$

together with initial conditions

$$
Q_{0}(0)=0, \quad U_{0}(0)=U_{b 0} \quad \text { and } \quad U_{0 y}(0)=0,
$$


and matching conditions

$$
U_{0}\left(L_{0}\right)=u_{c} \text { and } U_{0 y}\left(L_{0}\right)+Q_{0}\left(L_{0}\right) / \lambda_{c}=0 .
$$

Here we have used (3.4) with $q_{L}=0$ to determine the approximate form of $\alpha$, namely $\alpha \sim \mu-\mu^{3}$.

In [9] the existence of a nontrivial solution of this leading order problem is proved for all $0<\lambda<\lambda_{c}$. In order to assess the validity of the expansion procedure, it is valuable to compare it with a numerical solution of the full shooting problem for $\mu$ small. Using the numerical method discussed in $\S 6$, we solved shooting problem 2 for a succession of small values of $\mu$. We then estimated the exponents $a$ and $b$ in the expressions $L \propto \mu^{a}$ and $Q(L) \propto \mu^{b}$ by a local interpolation of this form. The results are in good agreement with the formal expansion predictions of $a=-\frac{1}{4}$ and $b=\frac{1}{4}$; for instance, we find that for $\mu=0.00123$ the numerically estimated values for $a$ and $b$ are -0.249 and 0.253 , respectively.

Form of solution for $\left(\lambda-\lambda_{c}\right)$ and $\mu \rightarrow 0$, and $L=0\left(\mu^{1 / 2}\right)$. We now examine the asymptotic form of solutions for which $\left(\lambda-\lambda_{c}\right)$ and $\mu \rightarrow 0$. For $\left(\lambda-\lambda_{c}\right) \rightarrow 0$ and $\mu$ of order unity we have seen that the solutions are characterised by small burning zones, whereas for $\mu \rightarrow 0$ and $\left(\lambda-\lambda_{c}\right)$ of order unity, they are characterised by burning zones of length $O\left(\mu^{-1 / 4}\right)$. Thus we expect that in the corner of parameter space $\lambda \sim \lambda_{c}, \mu \ll 1$ we will obtain different forms of solution depending upon our scaling of the length. In this section we examine the case $L=O\left(\mu^{1 / 2}\right)$, which is of particular interest because it allows us to determine analytically the end of the curve in $(\lambda, \mu)$ parameter space which divides regions in which $(U, U)$ and $(Q, U)$ switches operate.

Consider shooting problem 2 . We generalise the problem slightly to allow for the free boundary condition $Q(0)=\tau Q(L)=\tau c$. (See Remark 2 on shooting problem 2.) This corresponds to analysing ash with a nonzero heat capacity and is thus more realistic than the simplified case of $\tau=0$ considered so far. Note that $\tau \in[0,1)$.

We define $y=x / \mu^{1 / 2}$ and $L=L_{1} \mu^{1 / 2}$. Since we are analysing a bifurcating solution, we consider $L$ as given and determine $\lambda$, the bifurcation parameter, as $L$ varies.

Equations (3.9)-(3.11) give

$$
\begin{aligned}
& Q_{y}=\lambda \mu f(W), \\
& U_{y y}+\mu^{1 / 2} Q U_{y}+\mu(W-U)+\mu^{3 / 2} f(W)=0, \\
& \mu^{1 / 2} W_{y}=(U-W) .
\end{aligned}
$$

The initial conditions are

$$
\begin{aligned}
& Q(0)=\left(\mu-\alpha-\mu \alpha^{2}\right) /(1+\mu \alpha), \\
& U(0)=u_{b}, \\
& U_{y}(0)=\left(u_{b}-u_{a}\right) \alpha \mu^{1 / 2}, \\
& W(0)=u_{a}+\left(u_{b}-u_{a}\right) /(1+\mu \alpha) .
\end{aligned}
$$

The free boundary conditions, which determine $\alpha, u_{b}$ and $\lambda$ are

$$
\begin{aligned}
& Q(0)=\tau Q\left(L_{1}\right), \\
& U\left(L_{1}\right)=u_{c}, \\
& U_{y}\left(L_{1}\right)+\mu^{1 / 2}\left(u_{c}-u_{a}\right) Q\left(L_{1}\right)-\mu^{3 / 2}\left[W\left(L_{1}\right)-u_{a}\right]=0 .
\end{aligned}
$$


The appropriate form for the expansion is

$$
\begin{aligned}
& Q=\mu Q_{1}+\mu^{5 / 2} Q_{2}+\cdots, \\
& U=u_{c}+\mu^{3 / 2} U_{1}+\mu^{3} U_{2}+\cdots, \\
& U_{b}=u_{c}+\mu^{3 / 2} u_{b 1}+\mu^{3} u_{b 2}+\cdots, \\
& W=u_{c}+\mu^{3 / 2} W_{1}+\mu^{2} W_{2}+\cdots, \\
& \alpha=\alpha_{1} \mu+\alpha_{2} \mu^{5 / 2}+\cdots, \\
& \lambda=\lambda_{c}-\lambda_{c 1} \mu^{3 / 2}-\cdots,
\end{aligned}
$$

Substituting these expressions into the governing differential equations we obtain, to first order,

$$
\begin{aligned}
& Q_{1}=\left(1-\alpha_{1}\right)+\lambda_{c} f\left(u_{c}\right) y, \\
& U_{1}=W_{1}=u_{b 1}+\left(u_{c}-u_{a}\right) \alpha_{1} y-\frac{1}{2} f\left(u_{c}\right) y^{2} .
\end{aligned}
$$

From matching condition (5.7) we obtain

$$
u_{b 1}=\frac{1}{2} f\left(u_{c}\right) L_{1}^{2}-\left(u_{c}-u_{a}\right) \alpha_{1} L_{1} .
$$

Application of matching condition (5.6) gives us

$$
\left(1-\alpha_{1}\right)=\tau\left[\left(1-\alpha_{1}\right)+\lambda_{c} f\left(u_{c}\right) L_{1}\right]
$$

which rearranges to give

$$
\alpha_{1}=1-\frac{\tau \lambda_{c} f\left(u_{c}\right) L_{1}}{1-\tau} .
$$

From these results we deduce the following theorem.

THEOREM 5.2. Travelling wave solutions with a $(Q, U)$ switch exist in the regime $\mu \ll 1, \lambda \sim \lambda_{c}$ and $L=L_{1} \mu^{1 / 2}$ only for

$$
\frac{2}{\lambda_{c} f\left(u_{c}\right)}\left(\frac{1-\tau}{1+\tau}\right) \leqq L_{1} \leqq \frac{1-\tau}{\tau \lambda_{c} f\left(u_{c}\right)} .
$$

Proof. Since we require that $u_{b}>u_{c}$ in order that we have a $(Q, U)$ switch in operation (and shooting problem 2 is applicable) we must have $u_{b 1}>0$. From (5.9) this gives

$$
L_{1} \geqq \frac{2\left(u_{c}-u_{a}\right) \alpha_{1}}{f\left(u_{c}\right)} .
$$

Using (5.10) to eliminate $\alpha_{1}$, and noting that $\lambda_{c}=\left(u_{c}-u_{a}\right)^{-1}$, we obtain the lower bound on $L_{1}$.

Also, since all solutions must satisfy $\alpha_{1}>0$ in order that $Q(0)<\mu$ and (3.20) is satisfied, the upper bound on $L_{1}$ follows from (5.10).

Remarks on Theorem 5.2. (i) There will always be a region in parameter space in which $(Q, U)$ switch solutions exist, since

$$
\frac{2}{1+\tau}<\frac{1}{\tau} \text { for all } 0 \leqq \tau<1
$$

(ii) For $\tau=0$ the upper bound on $L_{1}$ disappears;

(iii) For $\tau \neq 0$ the proof of nonexistence of solutions in the asymptotic corner $\mu \ll 1$ and $\lambda \sim \lambda_{c}$ can be extended to all solutions which satisfy $L \gg L_{1} \mu^{1 / 2}$, again provided that $\tau>0$ and $a=0$. 
We now proceed to second order in the asymptotic expansion. The step-by-step asymptotic solution of the equation for $W$ is awkward because of the presence of a boundary layer of thickness $O\left(\mu^{1 / 2}\right)$ at $y=0$. Hence it is advantageous to integrate the linear equation for $W$ explicitly to determine $W$ as a function of $U$. This gives us

$$
\begin{aligned}
W(y) \sim u_{c} & +U_{1}(y) \mu^{3 / 2}-\alpha_{1} \exp \left\{-\mu^{-1 / 2} y\right\}\left(u_{c}-u_{a}\right) \mu^{2} \\
& -\left[\frac{d U_{1}}{d y}(y)-\exp \left\{-\mu^{-1 / 2} y\right\} \frac{d U_{1}}{d y}(0)\right] \mu^{2}+O\left(\mu^{5 / 2}\right) .
\end{aligned}
$$

The details of the second-order components of the solution provide little information of interest. However, we do evaluate $\lambda_{c 1}$. A straightforward evaluation of the matching condition (5.8) to $O\left(\mu^{3}\right)$, using (5.12) to avoid a full boundary layer analysis in the neighbourhood of $y=0$, gives us

$$
\lambda_{c}^{-1} \lambda_{c 1} f\left(u_{c}\right) L_{1}=\lambda_{c} f^{2}\left(u_{c}\right) L_{1}^{3} / 3-\alpha_{1} f\left(u_{c}\right) L_{1}^{2}+\alpha_{1}^{2} \lambda_{c}^{-1} L_{1} .
$$

In particular we may use this expression to calculate the locus of points dividing regions of $(U, U)$ switches and $(Q, U)$ switches. By (5.11) this locus of points is characterised by a value of

$$
L_{1}=\frac{2}{\lambda_{c} f\left(u_{c}\right)}\left(\frac{1-\tau}{1+\tau}\right) .
$$

Substituting this into (5.13) shows that, for $\mu \ll 1$, the locus of points dividing the two possible switching regimes is given by

$$
\lambda=\lambda_{c}-\left(\frac{1-\tau}{1+\tau}\right)^{2} \frac{\mu^{3 / 2}}{3 f\left(u_{c}\right)} .
$$

Form of solutions for $\left(\lambda \sim \lambda_{c}\right)$ and $\mu \rightarrow 0$ and $L=O\left(\mu^{1 / 2}\right)$; the $(U, U)$ switch case. In the previous part of this section we derived expressions for solutions of the $(Q, U)$ switch problem in the parameter regime $\left(\lambda-\lambda_{c}\right)$ and $\mu \rightarrow 0$ and $L=O\left(\mu^{1 / 2}\right)$. We also derived an asymptotic expression for the locus of points in $(\lambda, \mu)$ parameter space dividing regimes in which $(Q, U)$ and $(U, U)$ switches operate. Here we derive expansions for the $(U, U)$ switch case in the same parameter regime.

The differential equations and form of series expansion are the same as tor the $(Q, U)$ switch case. To first order we obtain

$$
\begin{aligned}
& Q_{1}=\left(1-\alpha_{1}\right)+\lambda_{c} f\left(u_{c}\right) y, \\
& U_{1}=\left(u_{c}-u_{a}\right) \alpha_{1} y-f\left(u_{c}\right) y^{2} / 2 .
\end{aligned}
$$

To ensure that $U_{1}\left(L_{1}\right)=0$ we require that

$$
\alpha_{1}=\lambda_{c} f\left(u_{c}\right) L_{1} / 2 \text {. }
$$

Note that a result analogous to Theorem 5.2 provides an upper bound on $L_{1}$ in order that we are in the regime of a $(U, U)$ switch, namely

$$
L_{1} \leqq \frac{2}{\lambda_{c} f\left(u_{c}\right)}\left(\frac{1-\tau}{1+\tau}\right) .
$$

As in the $(Q, U)$ switch case we can proceed to the second order. However we obtain no more useful information by doing this and so we omit the procedure.

6. Results of the numerical solution of the shooting problems. In this section we discuss results obtained from the numerical solution of shooting problems 1 and 2 . 
The numerical solution of two-point boundary value problems is discussed in a number of standard texts [2], [8]. To obtain the results in this section we use a shooting method combined with Newton-Kantorovich iteration.

By Theorem 4.1 we know that we can restrict our attention to the region $0<\lambda<\lambda_{c}$. Furthermore we know that a branch of nontrivial solutions is born at $\|U\|_{\infty} \sim u_{c}$ and $\lambda \sim \lambda_{c}$. Thus we may use this knowledge to provide an initial guess for a continuation procedure in the parameter $\lambda$. Using numerical solutions obtained in this manner we then perform continuation in the parameter $\mu$.

Figure 1 shows the bifurcation diagram $\left(\|U\|_{\infty}, \lambda\right)$ for the solution of shooting problems 1 and 2 . The free parameters are fixed at $\mu=5, u_{c}=0.9$ and $u_{a}=0.1$ and we take $f(W)=W^{2}$. The point $\lambda_{1}$ divides the regions in which a $(Q, U)$ switch operates (to the left) and a $(U, U)$ switch operates (to the right). Note that $d\left(\|U\|_{\infty}\right) / d \lambda$ is discontinuous there. $\lambda_{2}$ is the point at which constraint (3.20) ceases to be satisfied in the sense that $Q(L)<\mu$ for $\lambda>\lambda_{2}$. Thus, to the right of $\lambda=\lambda_{2}$ solutions to the travelling wave problem cease to exist. This concurs with the result of Theorem 5.1, which holds in the neighbourhood of $\lambda=\lambda_{c}$.

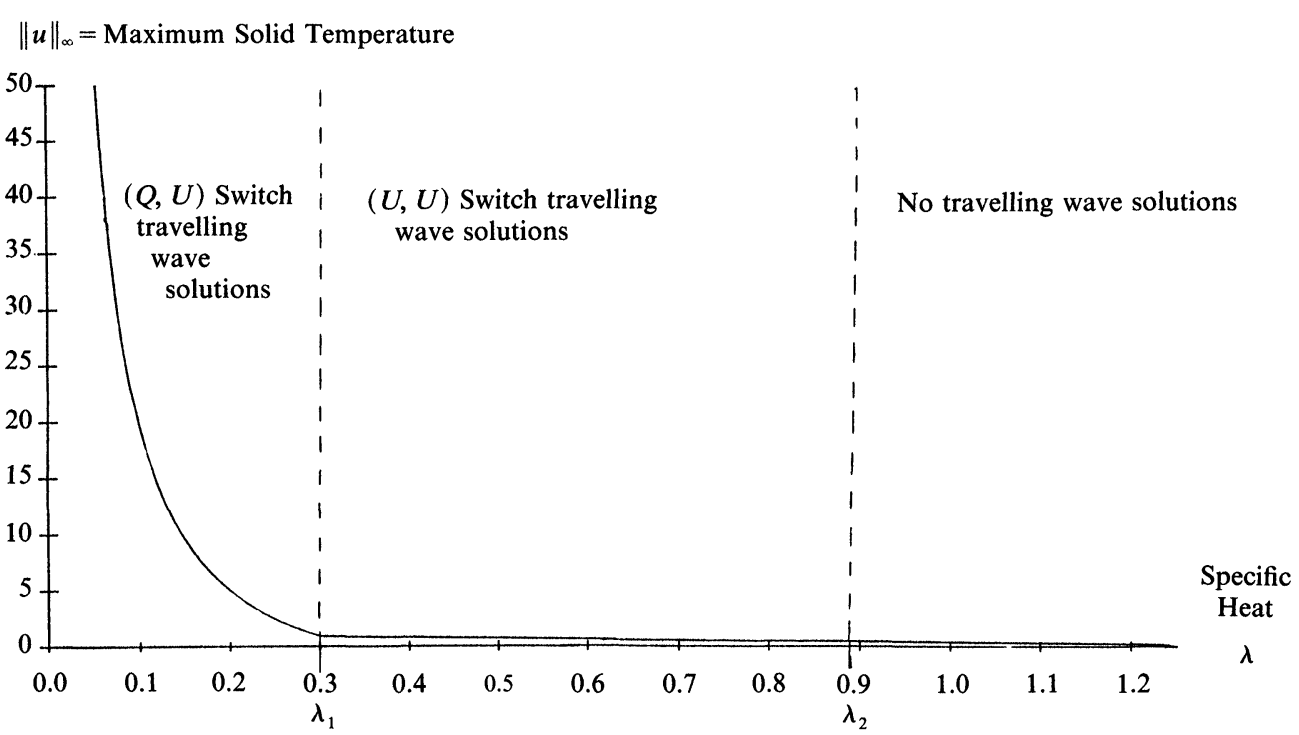

FIG. 1. Bifurcation diagram for shooting problems 1 and $2 ; \mu=5$.

Similar bifurcation diagrams are obtained for all values of $\mu$. In particular the fact that $\|U\|_{\infty}$ becomes unbounded as $\lambda \rightarrow 0$ is a common feature of all the solutions; this fact is a direct consequence of (4.1), which implies that the average of $U$ over $L$ is $O\left(\lambda^{-1}\right)$ as $\lambda \rightarrow 0$.

We now discuss the results obtained from continuation in the parameter $\mu$, for $\tau=0$. For $\mu \ll 1$ a $(Q, U)$ switch operates and the approximate form of the solution is given in $\S 5$. As $\mu$ increases the length of the burning zone decreases (see Fig. 2) until, at $\mu=\mu_{c}(\lambda)$, a minimum length is reached. As $L$ begins to increase again we reach a second critical value of $\mu=\mu_{*}(\lambda)$ at which $u_{b}=u_{c}$ and a $(U, U)$ switch takes over. Thereafter $L, c$ and $\|U\|_{\infty}$ continue to increase monotonically with $\mu$. However, $c / \mu$ decreases until finally, at $\mu=\mu^{*}(\lambda)$, a point is reached at which $c / \mu=1$. Hence constraint (3.20) is no longer satisfied and the solution to the travelling wave problem ceases to exist. 


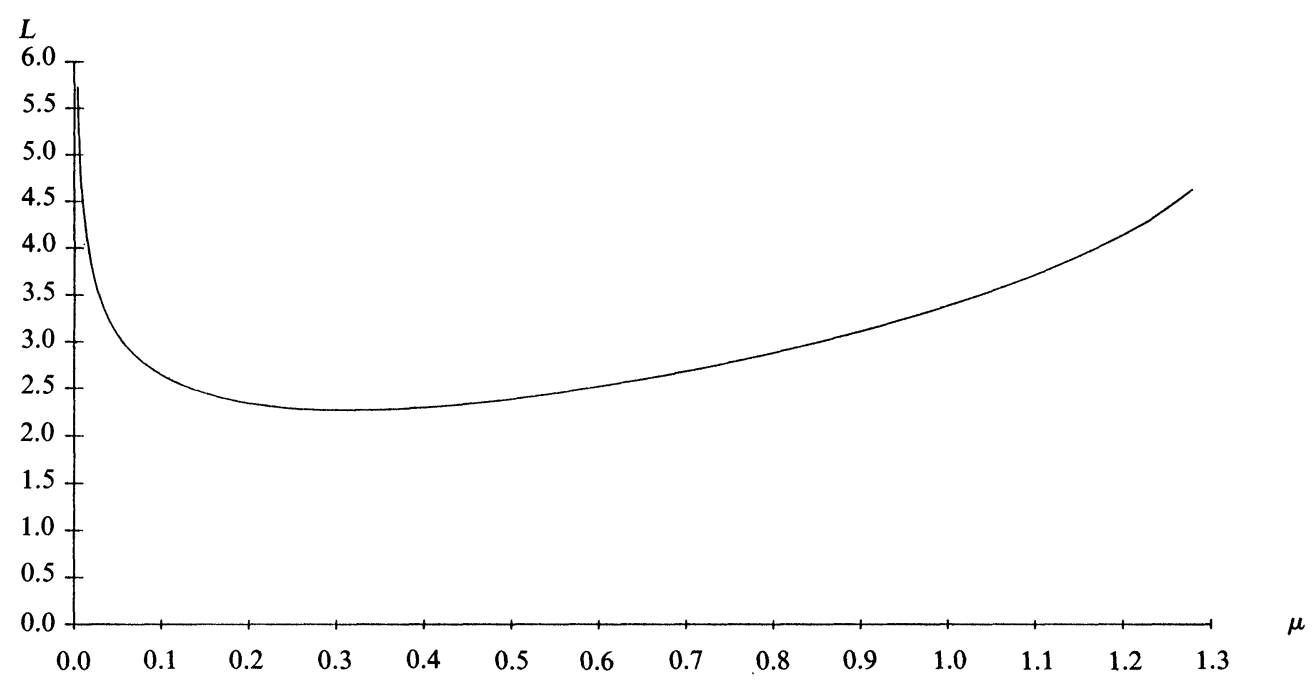

FIG. 2. Length of burning zone versus $\mu ;(Q, U)$ switch.

From this description we deduce the importance of the loci $\mu_{*}(\lambda)$ and $\mu^{*}(\lambda)$. We may find these numerically, again using a shooting method combined with NewtonKantorovich iteration. Notice that we have determined the ends of these loci analytically: $\mu_{*}(\lambda)$ is determined implicitly by (5.14) and $\mu^{*}(\lambda)$ is known to satisfy $\mu^{*}\left(\lambda_{c}\right)=1$, by Theorem 5.1. Again these local analytic expressions provide starting points for the numerical procedure.

The results of this section are summarised in Fig. 3, which shows the regions in $(\mu, \lambda)$ parameter space in which nontrivial travelling wave solutions exist. Figure 3 also delineates the regions in which $(Q, U)$ and $(U, U)$ solutions occur. Note that, for $\tau=0$, the lowest curve disappears since we can find solutions for arbitrarily small $\mu$ and $\lambda$.

Our numerical evidence supports the conjecture that when nontrivial travelling wave solutions exist they are unique, for our typical function $f(w)$. This conjecture is

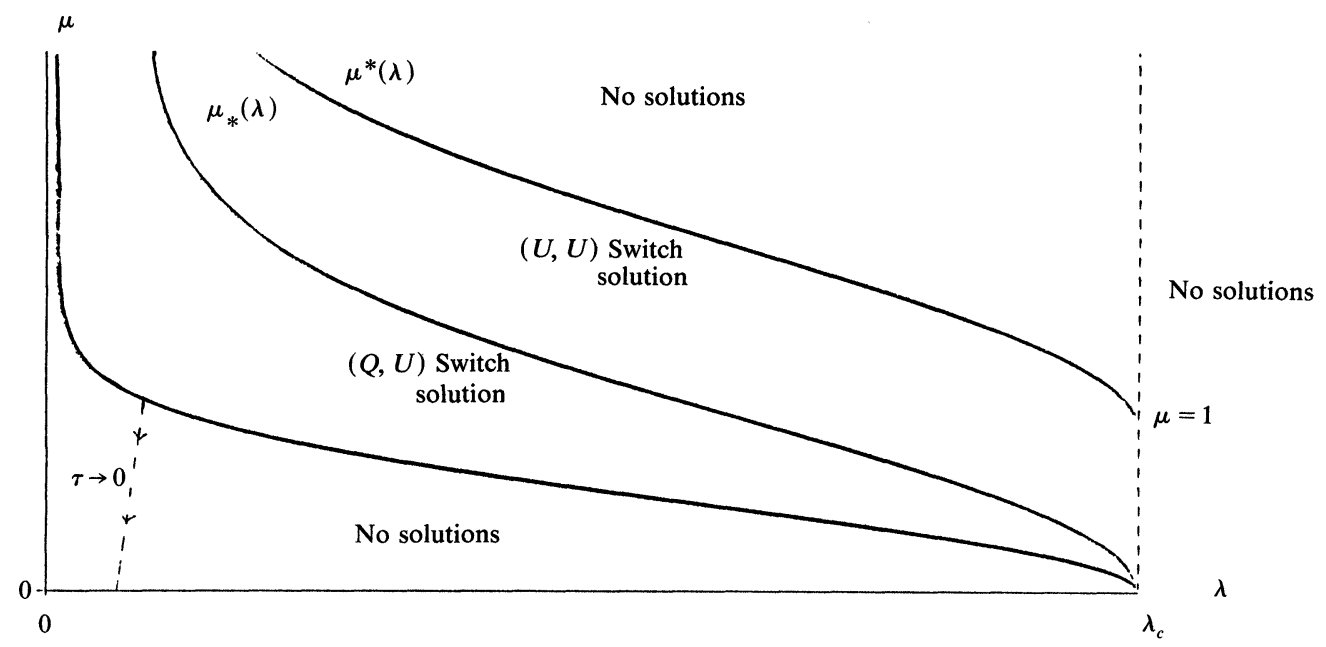

FIG. 3. Summary of existence theory for steady combustion waves. 
strengthened by the fact that there are no bifurcation points on the nontrivial solution branch in the neighbourhood of $\lambda=\lambda_{c}$. The proof of this result again employs series expansions to demonstrate that the Fréchet derivative of shooting problem 1, with respect to the nontrivial solution, is an invertible operator in the neighbourhood of $\lambda=\lambda_{c}$.

Throughout this paper we have been concerned with the case $a \equiv 0$ and so the variation of $g$, defined by (1.4), has been suppressed. The description of the solution behaviour will be modified for nonzero $a$, particularly for small values of the inlet velocity, proportional to $\mu$. For instance, we know from Theorem 5.2 that for $\tau \neq 0$ solutions of the travelling wave problem with $a=0$ cease to exist for $L$ sufficiently large, which corresponds to $\mu$ being sufficiently small. This nonexistence is associated with the suppression of the equation for $g$, which governs the depletion of oxygen during the combustion process. In practice, for small enough values of the inlet gas velocity, the depletion of oxygen eventually becomes significant, regardless of the initial concentration. Thus, for small enough values of $\mu$, we expect that a third switching regime will occur corresponding to oxygen exhaustion at the right-hand end the burning zone combined with solid exhaustion at the left-hand end.

\section{REFERENCES}

[1] S. N. Chow ANd J. Hale, Methods of Bifurcation Theory, Springer-Verlag, New York, 1982.

[2] H. B. Keller, Numerical Solution of Two-Point Boundary Value Problems, CBMS-NSF Regional Conference Series 24, Society for Industrial and Applied Mathematics, Philadelphia, PA, 1976.

[3] D. A. LAwson ANd J. Norbury, in Numerical Methods in Thermal Problems, Vol III, R. W. Lewis, et al., eds., Pineridge, Swansea, Wales, 1983.

[4] B. J. MATKowsky AND G. I. Sivashinsky, Propagation of a pulsating reaction front in solid fuel combustion, this Journal, 39 (1979), pp. 465-478.

[5] J. Norbury AND A. M. STUART, Models for porous medium combustion, Quart. J. Mech. and Appl. Math. 1987, submitted.

[6] —-, Parabolic equations with discontinuous forcing terms, Proc. Roy. Soc. Edinburgh Sect. A, 1986, submitted.

[7] — Travelling combustion waves in a porous medium. Part II-Stability, this Journal, 48 (1988), to appear.

[8] J. M. Roberts ANd J. S. Shipman, Two-Point Boundary Value Problems: Shooting Methods, Elsevier, New York, 1972.

[9] A. M. STUART, The existence of solutions of a two-point free boundary problem arising from the theory of porous medium combustion, IMA J. Appl. Math., to appear. 